\title{
Top multimedia information retrieval papers
}

\author{
Michael S. Lew ${ }^{1}$
}

Published online: 25 July 2016

(c) Springer-Verlag London 2016

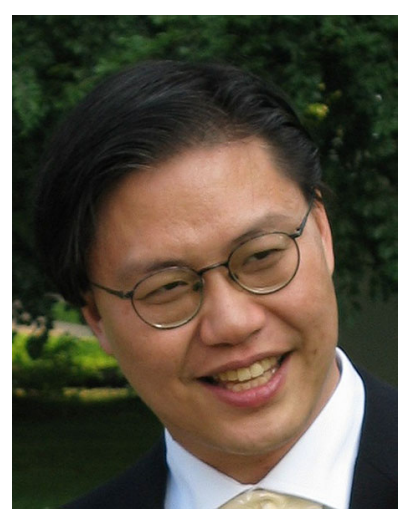

give a view on the important trends and challenges for the future.

\section{Visual related}

On-The-Fly Learning For Visual Search Of Large-Scale Image And Video Datasets

Ken Chatfield, Relja Arandjelović, Omkar Parkhi, Andrew Zisserman

http://link.springer.com/article/10.1007/s13735-015-0077-0 "The objective of this work is to visually search large-scale video datasets for semantic entities specified by a text query. The paradigm we explore is constructing visual models for such semantic entities on-the-fly, i.e. at run time, by using an image search engine to source visual training data for the text query".

From the first 4 years of the International Journal of Multimedia Information Retrieval (IJMIR), many new ideas have been contributed in diverse areas related to multimedia information retrieval. One of the most frequent requests from the readers is for the set of papers that were read (or downloaded) the most. Here it is:

The top ten most frequently read IJMIR papers below represents a mixture of new ideas for both multimedia search algorithms and feature representations. They were compiled directly from Springer statistics and are shown categorized by type of related media (unsorted within each category). The papers capture the state-of-the-art in important areas such as multimedia understanding, learning, eventrecognition and recommendation systems. Moreover, they

\footnotetext{
Michael S. Lew

mlew@liacs.nl

1 Leiden University, Leiden, The Netherlands
}

\section{High-Level Event Recognition In Unconstrained Videos} Yu-Gang Jiang, Subhabrata Bhattacharya, Shih-Fu Chang, Mubarak Shah

http://link.springer.com/article/10.1007/s13735-012-0024-2 "In this paper, we review current technologies for complex event recognition in unconstrained videos. While the existing solutions vary, we identify common key modules and provide detailed descriptions along with some insights for each of them, including extraction and representation of low-level features across different modalities, classification strategies, fusion techniques, etc".

\section{Bundle Min-Hashing}

Stefan Romberg, Rainer Lienhart http://link.springer.com/article/10.1007/s13735-013-0040-x "We present a feature bundling technique based on minHashing. Individual local features are aggregated with features from their spatial neighborhood into bundles. These 
bundles carry more visual information than single visual words".

\section{Interactive Search In Image Retrieval: A Survey}

Bart Thomee, Michael S. Lew

http://link.springer.com/article/10.1007/s13735-012-0014-4

"We highlight trends and ideas from over 170 recent research papers aiming to capture the wide spectrum of paradigms and methods in interactive search, including its subarea relevance feedback".

\section{Directional Local Extrema Patterns: A New Descriptor For Content Based Image Retrieval}

Subrahmanyam Murala, R. P. Maheshwari, R. Balasubramanian

http://link.springer.com/article/10.1007/s13735-012-0008-2

"In this paper, a new algorithm using directional local extrema patterns meant for content-based image retrieval application is proposed".

\section{A Study On Video Data Mining}

V. Vijayakumar, R. Nedunchezhian http://link.springer.com/article/10.1007/s13735-012-0016-2 "The objective of video data mining is to discover and describe interesting patterns from the huge amount of video data as it is one of the core problem areas of the data-mining research community".

\section{Special Issue On Concept Detection With Big Data}

Shih-Fu Chang, Thomas S. Huang, Michael S. Lew, Bart Thomee

http://link.springer.com/article/10.1007/s13735-015-0083-2

"By exploiting big data, the current generation of algorithms has contributed and developed both advances in accuracy and computational efficiency as well as new paradigms and techniques in concept detection".

\section{Music related}

Location-Aware Music Recommendation

Matthias Braunhofer, Marius Kaminskas, Francesco Ricci http://link.springer.com/article/10.1007/s13735-012-0032-2

"In recent years, a new research area of contextual music recommendation and retrieval has emerged. Context-aware music recommender systems are capable of suggesting music items taking into consideration contextual conditions, such as the user's mood or location, that may influence the user's preferences at a particular moment".

\section{Optical Music Recognition: State-Of-The-Art And Open} Issues

Ana Rebelo, Ichiro Fujinaga, Filipe Paszkiewicz, Andre R. S. Marcal, Carlos Guedes, Jaime S. Cardoso

http://link.springer.com/article/10.1007/s13735-012-0004-6

"This article provides an overview of the literature concerning the automatic analysis of images of printed and handwritten musical scores".

\section{Cross-media related}

Optimization Of Information Retrieval For Cross Media Contents In A Best Practice Network

Pierfrancesco Bellini, Daniele Cenni, Paolo Nesi http://link.springer.com/article/10.1007/s13735-014-0058-8 "Recent challenges in information retrieval are related to cross media information in social networks including rich media and web based content. In those cases, the cross media content includes classical file and their metadata plus web pages, events, blog, discussion forums, comments in multilingual". 